(RESEARCH ARTICLE)

\title{
Diagnostic and prognostic value of thrombocytopenia severity in Sudanese children with Falciparum malaria
}

Khalid Abdelsamea Mohamedahmed 1, Bakri Yousif Mohammed Nour 2, Adam Dawoud Abakar ${ }^{3}$ and Asaad Ma. Babker ${ }^{4}{ }^{*}$

\author{
${ }^{1}$ Department of Hematology and Immunology, Faculty of Medical Laboratory Sciences, University of Gezira, Wad Medani, \\ Sudan. \\ ${ }^{2}$ Department of Medical Parasitology, Blue Nile Institute for Communicable Diseases, University of Gezira, Wad Medani, \\ Sudan. \\ ${ }^{3}$ Department of Medical Parasitology, Faculty of Medical Laboratory Sciences, University of Gezira, Wad Medani, Sudan. \\ ${ }^{4}$ Department of Medical Laboratory Sciences, College of Health Sciences, Gulf Medical University, Ajman, UAE.
}

Publication history: Received on 10 June 2020; revised on 18 June 2020; accepted on 22 June 2020

Article DOI: https://doi.org/10.30574/wjarr.2020.6.3.0196

\begin{abstract}
Malaria remains one of the most significant global public health challenges, with more than 200 million clinical cases worldwide each year. Falciparum malaria accounting for up to $87.6 \%$ of malaria cases in Sudan. Platelets abnormalities as thrombocytopenia especially severe thrombocytopenia have been associated with mortality in patients with $P$. falciparum infection. The aim of this study was to study the relationships between thrombocytopenia and their severity with falciparum malaria severity, parasitemia and parasite count. This study was case-control hospital based study conducted among 200 Sudanese children admitted to Wad Medani Pediatric Hospital, Wad Medani, Sudan during period September to December 2018. The study population was divided into two groups. Group 1 (UM) was 100 children with uncomplicated falciparum malaria. Group 2 (SM) was 100 children with severe falciparum malaria were diagnosed by WHO criteria. A $2.5 \mathrm{ml}$ venous blood sample was collected from each children. The malaria parasitemia was determined from thick blood films using plus system. The parasite count (\% of parasitized red cells counting) was counted from thin blood film. Platelets count was determined using the Sysmex XP $300 \mathrm{~N}$ automated hematology analyzer (Sysmex, Kobe, Japan) and confirmed and assessed using stained thin blood film. The data were analyzed using SPSS software (V 20.0) and Stat disk software (V 13.0). The mean age and male: female ratio of Group 1 (UM) were $8.83 \pm 4.20$ years and 1:1.22 respectively and for Group $2(\mathrm{SM})$ were $8.63 \pm 3.40$ years and 1.56:1 respectively. Group 2 (61\%) associated with thrombocytopenia (mean PLTs $160.91 \pm 186.24 \times 10^{9} /$ ) more than Group 1 (25 \%) (mean PLTs $221.10 \pm 98.69 \times$ $\left.10^{9} / \mathrm{L}\right)(\mathrm{P}$ value $=0.000)$. The mild, moderate and severe thrombocytopenia account for $19 \%, 5 \%$ and $1 \%$ respectively among Group 1; and 22 \%, $28 \%$ and $11 \%$ respectively among Group 2 (P value 0.003). Malaria thrombocytopenia was significantly associated $(\mathrm{P}$ value $=0.000)$ and negatively correlated $(\mathrm{P}$ value $=0.000 ; \mathrm{r}=-0.341)$ with malaria parasitemia. The mean parasite count in malaria thrombocytopenia $(0.77 \pm 0.51 \%)$ was higher than malaria without thrombocytopenia $(0.53 \pm 0.36 \%)$ (P value 0.000$)$. Thrombocytopenia severity was significantly positive correlated with malaria parasitemia $(r=258 ; \mathrm{P}$ value $=0.017)$ and parasite count $(\mathrm{r}=0.229 ; \mathrm{P}$ value $=0.034)$. The study concluded that the severe thrombocytopenia associated commonly with severe falciparum malaria and falciparum malaria hyperparasitemia.
\end{abstract}

Keywords: Platelets count; Severe thrombocytopenia; Severe Falciparum malaria; Falciparum malaria hyperparasitemia; Sudanese children.

\footnotetext{
* Corresponding author: Asaad Ma. Babker
} 


\section{Introduction}

Falciparum malaria is the most virulent and pathogenic form of malaria (1), it is stills an important threat to public health in sub-Saharan Africa, and outside of Africa, particularly in young children, pregnant women and non-immune adults in communities with poor resources $(2,3)$. Falciparum malaria accounting for up to $80 \%$ of malaria cases globally (WHO, 2018) and 87.6\% in Sudan $(4,5)$.

Falciparum malaria associated with several hematological changes that involve the major blood cell lines such as red blood cells, white blood cells and platelets $(6,7)$, which play a significant role in severity of falciparum malaria $(8,9)$. Malaria hematological changes arising from hemolysis, host immune (inflammatory) response, bone marrow suppression, and splenic pooling (10).

Thrombocytopenia (platelet count less than $150 \times 10^{9} / \mathrm{L}$ ) is common platelets abnormalities and hematological changes $(7,11)$ as well as a common feature of malaria due to all plasmodium species particularly in falciparum malaria (occurs in up to $70 \%$ of falciparum malaria patients) $(11,12)$. Platelet survival is reduced to $2-4$ days in severe falciparum malaria $(7,13)$. The pathophysiology of malaria associated thrombocytopenia and reduced platelet survival rate are multifactorial. It has been associated with a variety of hematological insults arising from hemolysis, host inflammatory response, hematopoietic suppression, enhanced splenic uptake or sequestration and DIC (platelets may be removed from the circulation at sites of fibrin deposition) $(3,13)$. Some but not all studies have shown that there is strong association between thrombocytopenia and severity of malaria $(3,7,14)$. Severity and mortality of patients with falciparum malaria are increased with severe thrombocytopenia (3).

Previous studies have shown a consistent inverse correlation between parasitemia and the platelet count $(3,15-17)$. Patients with platelet counts $<150,000 / \mu \mathrm{L}$ are $12-15$ times more likely to have malaria infection than persons with platelet counts $>150,000 / \mu \mathrm{L}(7)$.

The present study aimed to study the relationships between thrombocytopenia and their severity with falciparum malaria severity, parasitemia and parasite count among Sudanese children.

\section{Methods}

This is study was case control hospital based study, conducted in Wad Medani Pediatric Hospital in collaboration with Faculty of Medical Laboratory Sciences, University of Gezira, Wad Medani, Sudan as part of a wider research project studying the association between TNF- $\alpha$ levels and TNF- $\alpha 238$ alleles polymorphisms and falciparum malaria anemia. The study population was divided into two groups. Group 1 (UM) was 100 subjects previously diagnosed as uncomplicated falciparum malaria by blood film or ICT. Group 2 (SM) was 100 subjects previously diagnosed as severe falciparum malaria by blood film and WHO criteria (18).

All study procedures were approved by the Ethics Committees of Ministry of Health, Gezira State and Faculty of Medical Laboratory Sciences, University of Gezira, Sudan. Informed consent was written from each participant parents.

A $2.5 \mathrm{ml}$ venous blood samples were collected by clean venipuncture in $\mathrm{K}_{3}$ EDTA containers. Thin and thick films were prepared immediately. Malaria parasitemia was determined from thick blood films using plus system (19). Parasite count (\% of parasitized red cells counting) was counted from thin blood film (18). Platelets count was determined using the Sysmex XP $300 \mathrm{~N}$ automated hematology analyzer (Sysmex, Kobe, Japan). The platelets count was confirmed and assessed using stained thin blood film. Thrombocytopenia was defined as a platelets count of less than $150 \times 10^{9} / \mathrm{L}(20)$. Mild thrombocytopenia was defined as a platelets count between 100 and $150 \times 10^{9} / \mu \mathrm{l}$, moderate thrombocytopenia was defined as between 50 and $99 \times 10^{9} / \mu \mathrm{l}$ and severe thrombocytopenia was defined as below $50 \times 10^{9} / \mu \mathrm{l}(20)$. The data were analyzed using SPSS software (V 20.0) and Stat disk software (V 13.0)..

\section{Results}

Study conducted on two groups; Group 1 (UM) (100 children with uncomplicated falciparum malaria) and Group 2 (SM) (100 children with severe falciparum malaria). The mean age of Groups 1 and 2 were $8.83 \pm 4.20$ years and $8.63 \pm 3.40$ years respectively $(\mathrm{P}$ value $=0.712)$. While the male: female ratio in Groups 1 and 2 were 1: 1.122 and 1.56: 1 respectively $(\mathrm{P}$ value $=0.023)$. Most cases in two Groups in age group between $6-10$ years $(41 \%$ in group $1 ; 47 \%$ in group 2). Most cases in Group 1 from rural area (70 \%), while approximately equal in Group 2 (P value $=0.002)($ Table $1)$. 
Table 1 Demographic characteristics of study participants.

\begin{tabular}{|c|c|c|c|}
\hline Factors & $\begin{array}{l}\text { Group } 1 \text { (UM) } \\
N=100\end{array}$ & $\begin{array}{l}\text { Group } 2(\mathrm{SM}) \\
\mathrm{N}=100\end{array}$ & P value $*$ \\
\hline \multicolumn{4}{|l|}{ Age (years) } \\
\hline Mean \pm SD & $8.83 \pm 4.20$ & $8.63 \pm 3.40$ & 0.712 \\
\hline \multicolumn{4}{|l|}{ Age group (years) } \\
\hline Less than 5 years & $24(24 \%)$ & $19(19 \%)$ & \\
\hline $6-10$ years & $41(41 \%)$ & $47(47 \%)$ & \\
\hline $11-15$ years & $29(29 \%)$ & $33(33 \%)$ & 0.185 \\
\hline More than 15 years & $6(6 \%)$ & $1(1 \%)$ & \\
\hline \multicolumn{4}{|l|}{ Gender } \\
\hline Male: Female ratio & $1: 1.22$ & $1.56: 1$ & 0.023 \\
\hline \multicolumn{4}{|l|}{ Residence } \\
\hline Rural & 70 & 49 & 0.002 \\
\hline Urban & 30 & 51 & \\
\hline
\end{tabular}

The hyperparasitemia (+++ and ++++ ) account for $93 \%$ in Group 2 and $32 \%$ among Group 1 (P value $=0.000)($ Table 1). Most cases in group 1 had parasite count < $0.5 \%$ (80\%) (mean of parasite count \% was $0.39 \pm 0.30 \%$ ), while most cases in group 2 (mean of parasite count \% was $0.88 \pm 0.42 \%)$ had parasite count $>0.5 \%(86 \%)(P$ value $=0.000)$ (Table 2).

The mean PLTs in Group 2 (160.91 $\left.\pm 186.24 \times 10^{9} / \mathrm{L}\right)$ was lower than Group $1\left(221.10 \pm 98.69 \times 10^{9} / \mathrm{L}\right)$, giving statistically highly significant differences between them (P value $=0.005$ ) (Table 2). PLTs had significant negative correlation within malaria parasitemia $(\mathrm{r}=-0.286$; $\mathrm{P}$ value $=0.000$; $)$ and malaria parasite count $(\mathrm{r}=-0.450$; $\mathrm{P}$ value $=$ 0.000) (Table 8).

Table 2 Clinical characteristics of study participants.

\begin{tabular}{|c|c|c|c|}
\hline Factors & $\begin{array}{l}\text { Group } 1 \text { (UM) } \\
N=100\end{array}$ & $\begin{array}{l}\text { Group } 2 \text { (SM) } \\
\mathrm{N}=100\end{array}$ & P value * \\
\hline \multicolumn{4}{|l|}{ Parasitemia } \\
\hline+ & $43(43 \%)$ & $4(4 \%)$ & \\
\hline++ & $25(25 \%)$ & $3(3 \%)$ & 0.000 \\
\hline+++ & $14(14 \%)$ & $21(21 \%)$ & \\
\hline++++ & $18(18 \%)$ & $72(72 \%)$ & \\
\hline Parasite count (\%) (Mean \pm SD) & $0.39 \pm 0.30$ & $0.88 \pm 0.42$ & 0.000 \\
\hline \multicolumn{4}{|l|}{ Parasite count (\%) frequency } \\
\hline$<0.5 \%$ & 80 & 16 & \\
\hline $0.5-1 \%$ & 13 & 54 & 0.000 \\
\hline$>1 \%$ & 7 & 30 & \\
\hline PLTs $\times 10^{9} /$ L $($ Mean \pm SD $)$ & $191.01 \pm 151.69$ & $290.91 \pm 97.77$ & 0.000 \\
\hline
\end{tabular}

Group 2 (61\%) associated with thrombocytopenia more than Group $1(25 \%)$ (P value = 0.000) (Figure 1). 


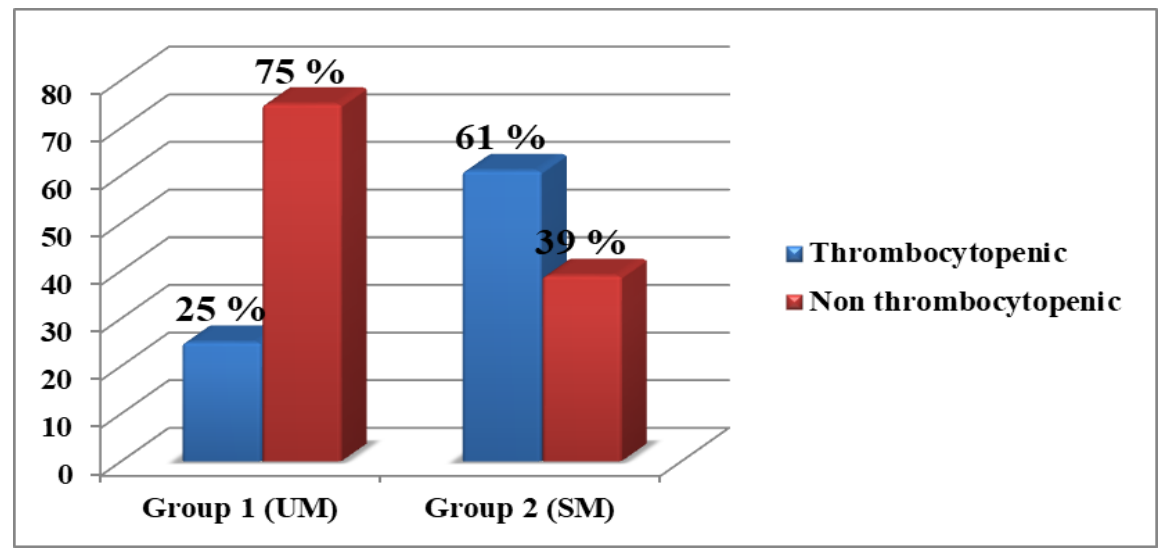

Figure 1 Association between malaria severity and malaria thrombocytopenia ( $\mathrm{P}$ value $=0.000)$.

Malaria thrombocytopenia account for $7 / 47$ in (+), 7/28 in (++), 21/35 in (+++), and 51/90 in (++++) giving highly significant association ( $\mathrm{P}$ value $=0.000$ ) (Table 3 ) and significant negative correlation $(\mathrm{P}$ value $=0.000 ; \mathrm{r}=-0.341$ ) (Figure 2) between malaria thrombocytopenia and parasitemia.

Table 3 Association between malaria parasitemia and malaria thrombocytopenia.

\begin{tabular}{llllll}
\hline Parameters & + & ++ & +++ & ++++ & P value * \\
& $\mathbf{N}=\mathbf{4 7}$ & $\mathbf{N}=\mathbf{2 8}$ & $\mathbf{N}=\mathbf{3 5}$ & $\mathbf{N = 9 0}$ & \\
\hline Thrombocytopenic & 7 & 7 & 21 & 51 & \\
Non-thrombocytopenic & 40 & 21 & 14 & 39 & 0.000 \\
\hline
\end{tabular}

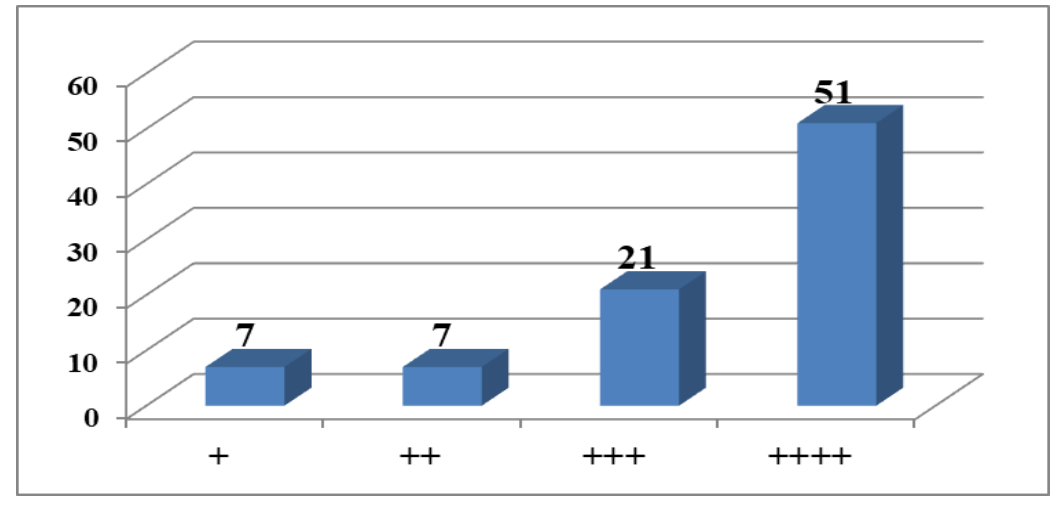

Figure 2 Correlation between malaria parasitemia and malaria thrombocytopenia $(P$ value $=0.000, r=-0.341)$.

The mean parasite count in children with malaria thrombocytopenia $(0.77 \pm 0.51 \%)$ was higher than children with malaria without thrombocytopenia $(0.53 \pm 0.36 \%)$, giving statistically highly significant differences between them $(\mathrm{P}$ value 0.000 ) (Table 4 ).

Table 4 Compare of parasite count \% between children with thrombocytopenia and without thrombocytopenia.

\begin{tabular}{llll}
\hline Parameters & $\begin{array}{l}\text { Thrombocytopenic } \\
\mathbf{N}=\mathbf{8 6}\end{array}$ & $\begin{array}{l}\text { Non-thrombocytopenic } \\
\mathbf{N}=\mathbf{1 1 4}\end{array}$ & P value $^{*}$ \\
\hline Parasite count $(\%)$ & $0.77 \pm 0.51$ & $0.53 \pm 0.36$ & 0.000 \\
\hline
\end{tabular}

The mild, moderate and severe thrombocytopenia account for $19 \%, 5 \%$ and $1 \%$ respectively among Group $1 ; 22 \%$, $28 \%$ and $11 \%$ among Group 2 (P value 0.003) (Table 5). 
Table 5 Association between malaria severity and of thrombocytopenia severity.

\begin{tabular}{llll}
\hline Thrombocytopenia severity & $\begin{array}{l}\text { Group 1 (UM) } \\
\text { N= 100 }\end{array}$ & $\begin{array}{l}\text { Group 2 (SM) } \\
\text { N= 100 }\end{array}$ & P value * \\
\hline Mild thrombocytopenia & 19 & 22 & 0.003 \\
Moderate thrombocytopenia & 5 & 28 & \\
Severe thrombocytopenia & 1 & 11 & \\
\hline
\end{tabular}

Despite the most mild, moderate and severe thrombocytopenia cases associated with malaria hyperparasitemia (+++ and ++++$)$, there were no statistically significant association between malaria parasitemia and thrombocytopenia severity $(\mathrm{P}$ value $=0.146)($ Table 6$)$, and there was significant positive correlation between them $(\mathrm{r}=258$; $\mathrm{P}$ value $=$ 0.017) (Table 8). Severe thrombocytopenia associated with malaria hyperparasitemia ( 4 with,+++ 8 with ++++ ) (Table 6).

Table 6 Association between malaria parasitemia and thrombocytopenia severity.

\begin{tabular}{llllll}
\hline Thrombocytopenia severity & + & ++ & +++ & ++++ & P value \\
& $\mathbf{N = 4 7}$ & $\mathbf{N = 2 8}$ & $\mathbf{N = 3 5}$ & $\mathbf{N = 9 0}$ & 0.146 \\
\hline Mild thrombocytopenia & 6 & 4 & 12 & 19 & 24 \\
Moderate thrombocytopenia & 1 & 3 & 5 & 8 & \\
Severe thrombocytopenia & 0 & 0 & $*$ P value $>0.05$ & &
\end{tabular}

Despite no differences in mean of parasite count \% between thrombocytopenia severity (P value $=0.514)($ Table 7$)$, there was significant positive correlation between them $(r=0.229$; P value $=0.034)$ (Table 8$)$.

Table 7 Compare of parasite count \% between thrombocytopenia severity.

\begin{tabular}{lllll}
\hline Parameters & Mild & Moderate & Severe & P value * \\
& $\begin{array}{l}\text { Thrombocytopenia } \\
\mathbf{N}=\mathbf{4 1}\end{array}$ & $\begin{array}{l}\text { Thrombocytopenia } \\
\mathbf{N}=\mathbf{3 3}\end{array}$ & $\begin{array}{l}\text { Thrombocytopenia } \\
\mathbf{N}=\mathbf{1 2}\end{array}$ & \\
\hline Parasite count $(\%)$ & $0.71 \pm 0.59$ & $0.81 \pm 0.47$ & $0.88 \pm 0.22$ & 0.514 \\
\hline
\end{tabular}

Table 8 Correlation between PLTs and thrombocytopenia severity with malaria parasitemia and parasite count.

\begin{tabular}{llll}
\hline Parameters & & Parasitemia & Parasite count \% \\
\hline Platelets count $\times 103 / \mu \mathrm{l}$ & Correlation coefficient & -0.286 & -0.205 \\
& P value* $^{*}$ & 0.000 & 0.004 \\
Thrombocytopenia & Correlation coefficient & 0.258 & 0.229 \\
severity & P value* & 0.017 & 0.034 \\
\hline \multicolumn{2}{r}{$*$ P value $>0.05$}
\end{tabular}

Most children with severe thrombocytopenia associated with severe falciparum malaria (11 out of 12, 91.7 \%), malaria hyperparasitemia ( 4 with,+++ 8 with ++++ ), and parasite count $>0.5 \%$ (11 out of $12,91.7 \%$ ).

\section{Discussion}

Falciparum malaria is still a major health problem in Sudan accounts for up to $80 \%$ of malaria cases globally (21) and about $87.6 \%$ of malaria cases in Sudan $(4,5)$. Poor sanitation and absence of majors protective is significantly leading 
to increased prevalence of the disease. Children suffer more malaria episodes and are more prone to severe malaria compared to adults and accounted for 61\% (266 000) of all malaria deaths. In fact, about 285,000 children died before their fifth birthdays in 2016 in Africa According to the World Health Organization (21). therefore malaria remains the largest cause of children death in Africa (22). The association of thrombocytopenia (Low Platelets count) with all plasmodium malaria types generally and falciparum malaria particularly is an equivocal. The current study aimed to throw a light on thrombocytopenia severity association with malaria severity, parasitemia and parasite count among Sudanese children.

Study conducted on two groups; Group 1 (UM): 100 children with uncomplicated (mean age $8.83 \pm 4.20$ years) falciparum malaria and Group 2 (SM): 100 children with severe falciparum malaria (mean age $8.63 \pm 3.40$ years). Similar studies were reported from different endemic areas in Africa (23-25).

In the present study male: female ratio was higher in Group 2 (1.56: 1) than Group 1 (1: 1.122) (P value = 0.023). Furthermore $81(40.5 \%)$ patients from Urban; while $119(59.5 \%)$ patients from rural (P value $=0.002)$. Similarly, survey was done in Sudan in 21,988 individuals to show the prevalence of malaria and results showed infection was higher in male more than female, and also higher in ruler area compare to urban (26).

The hyperparasitemia was most common Group 2 (93\%) compared to Group $1(32 \%)(\mathrm{P}$ value $=0.000)$.

Malaria thrombocytopenia was common in SM (62\%) compared to UM (25\%). The mild, moderate and severe thrombocytopenia were account $(31 \%, 30 \%$ and $3 \%$ respectively). Platelet survival is reduced to $2-4$ days in severe falciparum malaria (13). Gérardin et al reported that thrombocytopenia most common in SM (74.6\%) compared to UM (25.4\%) (17). Multiple studies in endemic area reported thrombocytopenia were observed in 73.6\% (27), 70\% (11), $85.5 \%$ of SM (28) of falciparum malaria children. The prospective study was conducted on 200 diagnosed cases of malaria. The data showed that 171 (85.5\%) patients were having low platelet count; 141 (70.5\%) had mild, 21 (10.5\%) moderate, and 9 (4.5\%) had severe thrombocytopenia. Twenty-nine (14.5\%) patients had normal platelet count (29). Study done in Ethiopia observed that the thrombocytopenia was found in $56.8 \%$, while mild, moderate, and severe thrombocytopenia accounted for $41.6 \%, 45.3 \%$ and $13.1 \%$ (23). Similar studies in endemic area found the severe thrombocytopenia was $8.8 \%$ in $45.6 \%$ of thrombocytopenia in France, $14.1 \%$ in $43.6 \%$ of thrombocytopenia in Senegal, $14.8 \%$ and in 58\% of thrombocytopenia in Libreville (Moulin et al., 2003), 5\% in 49\% of thrombocytopenia in Kenya (30), $1.5 \%$ in $48.6 \%$ of thrombocytopenia in Colombia (31), and $12.9 \%$ in $68.7 \%$ of thrombocytopenia in India (32). Similar previous studies observed that the thrombocytopenia was significantly more frequent and more profound in those with SM than in those with mild forms $(31,33)$.

Thrombocytopenia had strong negative correlation with parasitemia $(\mathrm{r}-0.335, \mathrm{P}$ value 0.000$)$, and parasite count $(\mathrm{r}-$ 0.268 , P value 0.000 ). Previous studies concluded that the thrombocytopenia is associated with peripheral parasitemia levels $(14,30,32,34-36)$. The average of platelets count among Group 2 was lower than Group 2 (P value 0.000$)$. The study done in Kenya showed the platelets count were significantly lower in malaria-infected children (30).

Children with thrombocytopenia were 24.4 times (odds ratio) more likely to have falciparum malaria. Maina et al., reported that children with platelets count of $<150,000 / \mathrm{uL}$ were 13.8 times (odds ratio) more likely to have malaria. Similar previous study concluded that Individuals with platelet counts $<150,000 / \mu \mathrm{L}$ are $12-15$ times more likely to have malaria infection than persons with platelet counts $>150,000 / \mu \mathrm{L}$. The risk of thrombocytopenia was $36.0 \%$ higher for SM (2.44 times) compared to UM, and 38.9\% higher for hyperparasitemia (3.08 times) compared to non hyperparasitemia. The risk of severe thrombocytopenia is 14.0\% higher for SM (4.50 times) compared to UM, and 16.2\% higher for hyperparasitemia compared to non hyperparasitemia. Previous study demonstrated that malaria parasite has a direct lytic effect on the platelets (33).

Thrombocytopenia is a frequent finding in falciparum malaria and possible causes are due to decreased platelets production, increased levels of cytokines and immunological destruction due to antibody and cellular immune responses, Oxidative stress damage of platelets, removal of platelets from circulation through enhanced splenic uptake and sequestration or by of fibrin deposition. In addition may result from antibodies, produced against the antimalarial drugs quinine and quinidine, interacting with platelets $(7,13,15)$.

\section{Conclusion}

The study concluded that the thrombocytopenia associated commonly with severe falciparum malaria and falciparum malaria hyperparasitemia particularly severe thrombocytopenia (PLTs $>50 \times 10^{9} / \mu$ ) ). So thrombocytopenia and their severity may help to assess the disease severity and to improve the management of falciparum malaria among patients. 


\section{Compliance with ethical standards}

\section{Acknowledgments}

The Wad Medani Pediatric Hospital and Faculty of Medical Laboratory Sciences is thanked for helping in laboratory working.

\section{References}

[1] Recker M, Bull PC and Buckee CO. (2018). Recent advances in the molecular epidemiology of clinical malaria. F1000Research, 7, 1159.

[2] World Health Organization. Severe and complicated malaria. 4th ed. Trop Med Int Health, (2014).

[3] Lampah DA, Yeo TW, Malloy M, Kenangalem E, Douglas NM, Ronaldo D, et al. (2015). Severe Malarial Thrombocytopenia: A Risk Factor for Mortality in Papua, Indonesia. The Journal of Infectious Diseases, 211, 62334.

[4] Roll Back Malaria in Sudan. Sudan Malaria Treatment Protocol (2017). Federal Ministry of Health, 8-40.

[5] Mohamedahmed KA, Mustafa RE, Abakar AD and Nour BYM. (2019). Evaluation of Neutrophil Lymphocyte Ratio (NLR) in Sudanese Children with Falciparum Malaria. IJAHMR, 3(5), 1-6.

[6] Antwi-Baffour S, Kyeremeh R, Buabeng D, Adjei JK, Aryeh C and Kpentey G. (2018). Correlation of malaria parasitaemia with peripheral blood monocyte to lymphocyte ratio as indicator of susceptibility to severe malaria in Ghanaian children. Malar J, 17, 419.

[7] Erhabor 0, Otunuya 0, Erhabor T, Knox VD and Adias TC. (2018). Anaemia and Thrombocytopenia among Children of African Descent with Acute Plasmodium Falciparum Malaria in Sokoto, North Western Nigeria, 4(1), 051.

[8] Prasad PL, Rai PL and Hussain MS. (2018). A study of haematological profile of malaria in a tertiary care centre of western Uttar Pradesh, India. Int J Contemp Pediatr, 5, 1115-1119.

[9] Mohamedahmed KA, Abakar AD, Ahmed MO, Mukhtar MM and Nour BYM. (2019). The Role of TNF- $\alpha$ Levels as Predictive Diagnostic Biomarker Among Children with Severe Falciparum Malaria in Endemic Area in Sudan. IJAHMR, 3(7), 1-6.

[10] Menendez C, Fleming AF and Alonso PL. (2000). Malaria-related anaemia. Parasitol Today, 16, $469-476$.

[11] Suh KN, Kain KC, Keystone JS and Malaria. (2004).Canadian Medical Association Journal, 170(11), $1693-1703$.

[12] Lacerda MV, Mourao MP, Coelho HC and Santos JB. (2011). Thrombocytopenia in malaria: who cares?. Mem Inst Oswaldo Cruz, 106(suppl 1), 52-63.

[13] Warrell DA. (2002). Clinical features of malaria. In: Warrell DA; Gilles HM editors. Essential Malariology. 4th ed. Arnold, a member of the Hodder Headline Group, London, 191-205.

[14] Ladhani S, Lowe B, Cole AO, Kowuondo K and Newton CRJC. (2002).Changes in white blood cells and platelets in children with falciparum malaria: relationship to disease outcome. Brit J Haematol, 119, 839-847.

[15] Leal-Santos FA, Silva SB, Crepaldi NP, et al. (2013). Altered platelet indices as potential markers of severe and complicated malaria caused by Plasmodium vivax: a cross-sectional descriptive study. Malar J, 12, 462.

[16] Kotepui M, Piwkham D, PhunPhuech B, Phiwklam N, Chupeerach C and Duangmano S. (2015). Effects of Malaria Parasite Density on Blood Cell Parameters. PLoS ONE, 10(3), e0121057.

[17] Gérardin P, Ka AS and Imbert P. (2018). Thrombocytopenia as Additional Marker of Severity in African Children with Plasmodium Falciparum Malaria. J Infect Dis Pathog, 1(1), 1-5.

[18] World Health Organization. (2015). Guidelines for The Treatment of Malaria. 3rd edition. WHO in Geneva.

[19] World Health Organization.(2010). Basic Malaria Microscopy. 2nd edition. WHO in Geneva.

[20] Bain BJ, Bates I, Laffan M and Lewis M. (2011). Dacie and Lewis Practical Hematology. (11th ed). British: Elsevier Ltd, 394-397.

[21] World Health Organization. (2018). 11th World malaria report. WHO in Geneva. 
[22] Roberts D and Matthews G. (2016). Risk factors of malaria in children under the age of five years old in Uganda. Malar J, 15, 246.

[23] Birhanu M, Asres Y, Adissu W, Yemane T, Zemene E and Gedefaw L. (2017). Hematological Parameters and Hemozoin-Containing Leukocytes and Their Association with Disease Severity among Malaria Infected Children: A Cross-Sectional Study at Pawe General Hospital, Northwest Ethiopia. Interdisciplinary Perspectives on Infectious Diseases, 7.

[24] Frimpong A, Kusi KA, Tornyigah B, Ofori MF and Ndifon W. (2018). Characterization of T cell activation and regulation in children with asymptomatic Plasmodium falciparum infection. Malar J, 17, 263.

[25] Madukaku CU, Chimezie OM, Chima NG, Hope O and Simplicius DIN. (2015). Assessment of the haematological profile of children with malaria parasitaemia treated with three different artemisinin-based combination therapies. Asian Pac J Trop Dis, 5(6), 448-453.

[26] National Malaria Control Programme. (2010). Five Years Strategies Plan for the National Malaria Control Programme, Sudan 2011 - 2015. Federal Ministry of Health.

[27] Sahoo A and Das K. (2013). Coagulation profile in Sever Falciparum Malaria and it is clinical correlation. International Journal of General Medicine and Pharmacy, 2(1), 2-14.

[28] Prasad R, Das BK, Pengoria R, Mishra OP, Shukla J, and Singh TB. (2009). Coagulation Status and Platelet Functions in Children with Severe Falciparum Malaria and their Correlation of Outcome. J Trop Pediatr, 55, 374-378.

[29] Shaikh MA, Shakeel A, Inayat UD and Dur EY. (2011). Platelet count in malaria patients. J Ayub Med Coll Abbottabad, 23(1), 143-145.

[30] Maina RN, Walsh D, Gaddy C, Hongo G, Waitumbi J, Otieno L, et al. (2010). Impact of Plasmodium falciparum infection on haematological parameters in children living in Western Kenya. Malaria Journal, 9(3), S4.

[31] AreÂvalo-Herrera M, Rengifo L, Lopez-Perez M, Arce-Plata MI, Garcõ̂̂a J and Herrera S. (2017). Complicated malaria in children and adults from three settings of the Colombian Pacific Coast: A prospective study. PLoS ONE, 12(9), e0185435.

[32] Chhawchharia R, Kolhe S, George R and Lahiri KR. (2016). Clinical and Hematological Changes in Childhood Malaria in India. IOSR Journal of Dental and Medical Sciences, 15(7), 86-90.

[33] Gerardin P, Rogier C, Ka AS, Jouvencel P, Brousse V and Imbert P. (2002). Prognostic Value of Thrombocytopenia in African Children with Falciparum Malaria. Am. J. Trop. Med. Hyg, 66(6), 686-691.

[34] Wassmer SC, Taylor TE, Rathod PK, Mishra SK, Mohanty S, Arevalo-Herrera M, et al. (2015). Investigating the Pathogenesis of Severe Malaria: A Multidisciplinary and Cross-Geographical Approach. Am. J. Trop. Med. Hyg, 93(3), 42-56.

[35] Deroost K, Pham TT, Opdenakker G and Van den Steen PE. (2016). The immunological balance between host and parasite in malaria. FEMS Microbiology Reviews, 40(2), 208-257.

[36] Abdelnassir MA, Hafiz AH, Gad Allah Mn and Mohammed SE. (2019). Hematological Changes in Sudanese Patients with Falciparum Malaria Attending Elnihoud Teaching Hospital. Sudan Journal of Medical Sciences, 14(1), 24-30.

[37] Moulin F, Lesage F, Legros A-H, Maroga C, Moussavou A, Guyon P, et al. (2003). Thrombocytopenia and Plasmodium falciparum malaria in children with different exposures. Arch Dis Child, 88, 540-541.

\section{How to cite this article}

Khalid AM, Bakri Yousif MN, Adam DA and Asaad MB. (2020). Diagnostic and prognostic value of thrombocytopenia severity in Sudanese children with Falciparum malaria. World Journal of Advanced Research and Reviews, 6(3), 197208. 